\title{
Macrophage-Mediated Dorsal Root Ganglion Damage Precedes Altered Nerve Conduction in SIV-Infected Macaques
}

\author{
Victoria A. Laast, ${ }^{* \dagger}$ Beom Shim, ${ }^{\ddagger}$ \\ Lisa M. Johanek, ${ }^{\ddagger}$ Jamie L. Dorsey, ${ }^{*}$ \\ Peter E. Hauer, ${ }^{\S}$ Patrick M. Tarwater," \\ Robert J. Adams, ${ }^{\star}$ Carlos A. Pardo, ${ }^{\dagger \S}$ \\ Justin C. McArthur, ${ }^{\S}$ Matthias Ringkamp, ${ }^{\ddagger}$ and \\ Joseph L. Mankowski*†§ \\ From the Departments of Molecular and Comparative \\ Pathobiology, ${ }^{*}$ Patbology, ${ }^{\dagger}$ Neurosurgery, ${ }^{\ddagger}$ and Neurology, Johns \\ Hopkins University School of Medicine, Baltimore, Maryland; \\ and the Division of Biostatistics and Epidemiology, "I Paul L. \\ Foster School of Medicine, El Paso, Texas
}

Peripheral neuropathy is the most common neurological complication of HIV-1 infection, affecting over onethird of infected individuals, including those treated with antiretroviral therapy. To study the pathogenesis of HIV-induced peripheral nervous system disease, we established a model in which SIV-infected macaques developed changes closely resembling alterations reported in components of the sensory pathway in HIVinfected individuals. Significant declines in epidermal nerve fiber density developed in SIV-infected macaques, similar to that of $\mathrm{HIV}$-infected individuals with neuropathy. Changes in dorsal root ganglia (DRG) included macrophage infiltration, SIV replication in macrophages, immune activation of satellite cells, and neuronal loss. To determine whether dorsal root ganglion damage was associated with altered nerve function, we measured unmyelinated $C$-fiber conduction velocities (CV) in nerves of SIV-infected macaques and compared CV changes with DRG alterations. Twelve weeks postinoculation, SIV-infected macaques had significantly lower C-fiber conduction velocity in sural nerves than uninfected animals and the magnitude of conduction velocity decline correlated strongly with extent of DRG macrophage infiltration. Thus, injury to neurons in the DRG - mediated by activated macrophages - preceded altered conduction of unmyelinated nerve fibers in SIV-infected macaques, suggesting that macrophage-mediated DRG damage may be the initiating event in HIV-induced sensory neuropathy. (Am J Patbol 2011, 179:2337-2345; DOI: 10.1016/j.ajpath.2011.07.047)

Peripheral neuropathy is currently the most common neurological complication associated with HIV-1 infection with over one-third of infected individuals exhibiting signs of peripheral neuropathy. ${ }^{1,2}$ The most typical clinical manifestation of HIV-induced peripheral nervous system disease is a debilitating syndrome called distal sensory polyneuropathy (DSP) with gradual onset of bilateral pain described as aching, painful numbness or burning that is most severe in the soles of the feet. ${ }^{3,4}$ Although not life threatening, HIVinduced peripheral neuropathy markedly affects the quality of life of many HIV-infected patients. ${ }^{1,5}$

The effects of antiretroviral therapy on HIV-induced peripheral nervous system (PNS) disease remain to be determined. Although the incidence of HIV neuropathy may decrease with antiretroviral treatment, many treated HIVinfected individuals still suffer from HIV-induced PNS disease. It is well known that treatment of HIV infection with nucleoside analogues as well as protease inhibitors to control systemic viral replication appears to induce unintended toxic neuropathy as a side effect, thereby further complicating clinically based studies of the pathogenesis of HIVinduced PNS disease. ${ }^{1,6}$ For these reasons, estimates of the incidence of HIV neuropathy in highly active antiretroviral therapy-treated individuals vary widely (from 13\% to $52 \%$ ) depending on the cohort evaluated. ${ }^{3,7-9}$

Although HIV-induced PNS disease exhibits clinical features consistent with length-dependent nerve damage, the pathogenesis of HIV-induced damage to the sensory nociceptive pathway is poorly understood, and

Supported by NIH grants NS055651 (J.L.M.), RR07002 (V.A.L.), NS44807, and U54NS043011 (J.C.M.); animal studies were also supported by NIH MN61189.

Accepted for publication July 11, 2011.

Current address of V.A.L., Covance Laboratories, Chantilly, VA.

Address reprint requests to Joseph L. Mankowski, D.V.M., Ph.D., Johns Hopkins University School of Medicine, Department of Molecular and Comparative Pathobiology, 733 N. Broadway, 835 Broadway Research Bldg., Baltimore, MD 21205-2196. E-mail: jmankows@jhmi.edu. 
the site of primary damage in HIV-induced PNS disease remains to be established. Damage to the cell bodies of sensory neurons located in the dorsal root ganglia (DRG) may be the initiating event leading to HIV neuropathy, with neuronal damage resulting in a secondary dyingback process including axonal degeneration. ${ }^{10}$ Another possibility is that the primary pathological change is a distal axonopathy resulting from primary nerve fiber damage.3,11 Distinguishing between damage to these two regions is difficult to study in HIV-infected individuals because of our inability to repeatedly sample different components of the PNS throughout infection.

It is not clear whether HIV-induced peripheral neuropathy is a consequence of persistent release of neurotoxic products by activated macrophages (including the cytokines tumor necrosis factor- $\alpha$ or IL-1 $\beta$ ), of neurotoxic viral proteins such as HIV glycoprotein (gp)41, gp120, or Tat, or of the combined effects of macrophage activation and viral proteins. ${ }^{12}$ Pathogenesis studies are complicated by the treatment of the majority of HIV-infected individuals with antiretroviral therapy. Animal models are thus essential to determine where and when initial neuronal injury occurs and to dissect out the effects of HIV infection separate from the effects of potentially neurotoxic antiretroviral drugs. ${ }^{13,14}$ It is crucial to define the mechanisms underlying HIV PNS disease because this knowledge would direct appropriate therapeutic strategies.

To study the pathogenesis of HIV-induced PNS disease, we established an animal model in which SIV-infected macaques developed PNS changes closely resembling alterations seen in HIV-infected individuals, including macrophage infiltration, SIV replication in macrophages, and neuronal loss in sensory ganglia. In our previous studies, we documented alterations in the trigeminal ganglia of SIVinfected macaques, demonstrating that replication of SIV in macrophages of sensory ganglia closely resembles the alterations reported in the DRG from HIV-infected individuals. ${ }^{15}$ In this report, we have extended our studies of SIVinfected macaques to define the relationship between damage to critical components of the sensory pathway and the corresponding alterations in nerve function. This study specifically examined morphological alterations in two key components of the peripheral sensory pathway, the lumbar dorsal root ganglia and the epidermal nerve fibers that are connected by the small unmyelinated C-fibers that course in peripheral nerves. To determine whether altered peripheral nerve function closely corresponded with either sensory ganglia alterations or changes in epidermal nerve fiber density, we measured unmyelinated C-fiber conduction velocities (CV) in sural nerves of SIV-infected macaques and then compared changes in sural nerve conduction properties with corresponding lumbar DRG and epidermal nerve fiber alterations.

\section{Materials and Methods}

\section{Animal Studies}

Twenty-eight pigtailed macaques were inoculated intravenously simultaneously with the neurovirulent clone SIV/
17E-Fr and the immunosuppressive swarm SIV/ DeltaB670 and then euthanized at either 6 weeks $(n=$ $11), 8$ weeks $(n=10)$, or 12 weeks postinoculation (p.i.) $(n=7) .{ }^{16}$ This particular SIV/macaque model has been previously characterized in detail in studies of SIV central nervous system disease pathogenesis; SIV-infected animals uniformly progress to AIDS with comparable high plasma viral loads within 12 weeks p.i. ${ }^{17,18}$ Six additional age-matched uninfected pigtailed macaques served as uninoculated, virus-negative controls. Lumbar (L3 to L6) dorsal root ganglia that contain the cell bodies of sensory neurons innervating the skin of distal hind limbs and sural and sciatic nerves were harvested from all pigtailed macaques. The animal procedures in this study were performed according to the principles set forth by the Institutional Animal Care and Use Committee at Johns Hopkins University and the National Research Council's Guide for the care and use of laboratory animals.

\section{Measurements of Epidermal Nerve Fiber Density}

To measure epidermal nerve fiber (ENF) density in the skin of the plantar footpad surface of the hind limb, 3-mmdiameter punch footpad samples were obtained at necropsy from the identical locale. Footpad skin sections were fixed for 12 to 24 hours in $2 \%$ paraformaldehyde/ lysine/periodate fixative at $4^{\circ} \mathrm{C}$, rinsed with $0.08 \mathrm{~mol} / \mathrm{L}$ Sorensen's phosphate buffer, and then transferred to cryoprotective buffer $(20 \%$ glycerol in $0.08 \mathrm{~mol} / \mathrm{L}$ Sorensen's phosphate buffer) until processed as previously described. ${ }^{19}$ Cryoprotected samples were sectioned at a thickness of $50 \mu \mathrm{m}$ on a sliding microtome and then immunostained for PGP9.5, a panaxonal marker (1:2000; Chemicon, Temecula, CA), as previously described. ${ }^{20,21}$ ENF density was measured using a modification of the method used by Kennedy et $\mathrm{al}^{13}$ and McCarthy et al. ${ }^{20}$ Briefly, 15 adjacent, nonoverlapping collapsed Z-stack images were obtained for each PGP9.5 immunostained skin section. Serial Z-stack images for each microscopic field were collected at $0.5-\mu \mathrm{m}$ intervals using $\times 400 \mathrm{mag}$ nification on a Zeiss microscope equipped with a z-motor (Carl Zeiss, Oberkochen, Germany). PGP9.5 immunoreactivity in the collapsed Z-stack images was then measured by digital image analysis using iVision software (BioVision Technologies, Exton, PA). ${ }^{17}$ Results were normalized to the thickness of each skin sample (the $z$ distance of the stack) for each image to control for any variations in the thickness of immunostained skin sections.

\section{Immunohistochemistry}

Immunohistochemistry was performed on Streck-fixed (Streck tissue fixative; Streck Laboratories, Omaha, NE), paraffin-embedded sections of dorsal root ganglia from SIV-infected and control animals. Tissue sections were deparaffinized in changes of Histo-Clear (National Diagnostics, Atlanta, GA) and then rehydrated in a gradient series of alcohol. After antigen retrieval in sodium citrate buffer for 8 minutes, sections were washed and then blocked against endogenous peroxidase followed by incubation in the appropriate antibody dilution (KK41 
1:4000; NIH AIDS Research and Reference Reagent Program; CD68, 1:2000; clone KP1 and GFAP, 1:4,00, Z0334; DAKO, Carpinteria, CA) for an hour at room temperature. Sections were then incubated sequentially in biotinylated secondary multilink antibody and horseradish peroxidase-labeled streptavidin (Biogenex, San Ramon, CA). The chromogen reaction was subsequently detected by incubating the sections in substrate 3,3'diaminobenzidine. The washed and cleared tissue sections were then coverslipped with Permount mounting medium (Fisher Scientific, Pittsburgh, PA). The amount of immunostaining for the macrophage marker CD68 and the satellite cell activation marker GFAP was measured by digital image analysis as described previously. ${ }^{17}$ Twenty nonoverlapping adjacent fields at $\times 200$ magnification were captured with a Retiga 2000R digital camera (Qlmaging, Surrey, BC, Canada) mounted on a Nikon E600 microscope (Nikon, Tokyo, Japan). Binarized images were then analyzed using iVision imaging software (BioVision Technologies, Exton, PA). The mean total area occupied by immunopositive pixels in the DRG was then calculated for each animal.

\section{Viral Load in DRG}

To measure SIV RNA levels, real-time RT-PCR was performed on RNA isolated from DRG. At postmortem, samples were snap frozen in liquid nitrogen for RNA isolation as previously described. ${ }^{20}$ RNA was isolated from $25 \mathrm{mg}$ of tissue using the RNA STAT-60 Kit (Tel-Test, Inc., Friendswood, TX) and an RNeasy Mini Kit (Qiagen, Valencia, CA). Quantitation of SIV RNA in extracted brain tissue was done with SIV gag region primers and probe. Real-time RT-PCR detected both cell-associated full-length viral transcripts and genomic RNA present in tissue-associated virions.

\section{Confocal Microscopy for Colocalization of SIV and Macrophages}

To perform colocalization studies using confocal microscopy, 7- $\mu \mathrm{m}$-thick sections of paraffin-embedded DRG on gelatin-subbed glass slides were deparaffinized and rehydrated. Sections were microwaved in sodium citrate buffer for 8 minutes to expose antigen epitopes, following which, sections were washed and then blocked in 3\% normal goat serum containing $1 \%$ triton for an hour at room temperature. Incubation of sections in appropriate antibody dilution (KK41 1:4000; NIH AIDS Research and Reference Reagent Program; Iba-1 1:250; WAKO Lab Chemicals, Richmond, VA) was performed for 48 hours at $4^{\circ} \mathrm{C}$. Sections were then incubated in fluorophore-tagged secondary antibodies (Cy3-conjugated goat anti-rabbit IgG, 1:2000; Jackson ImmunoResearch Lab Inc, West Grove, PA), AlexaFlour-488-conjugated goat anti-mouse IgG (1:1000; Molecular Probes, Eugene, OR) for 2 hours at room temperature in the dark. Sections were subsequently washed, coverslipped with Mowiol mounting medium, and visualized in a single $X: Y$ plane using a Nikon C1 confocal laser microscopy system mounted on a Nikon Eclipse TE2000-E microscope.

\section{Quantitation of Neuronal Density in DRG}

Neuronal density in L5 DRG was measured in H\&E-stained sections using the unbiased stereological method of area fractionation via the Stereo Investigator fractionator program (MicroBrightField Inc, Colchester, VT). ${ }^{22-24}$ Between 35 and 40 counting frames were systematically and randomly placed within the delineated area of each DRG section. Each counting grid contained a group of 20 crosses, and for each sample, the sum of the crosses covering neurons was divided by the total number of grid crosses sampled to estimate the fraction of the area (fractional area) occupied by neurons. ${ }^{22}$

\section{Electrophysiology Studies to Measure C-Fiber Conduction Velocity}

To examine whether SIV infection induced functional alterations in peripheral nerves, nerve conduction studies were performed on isolated sural nerves obtained from SIV-infected pigtailed macaques and from uninfected control macaques $(n=6)$ for comparison. To measure the conduction velocity of small sensory C-fibers, a $6-\mathrm{cm}$ long segment of mid-sural nerve was harvested from the same location (lower limb, calf region) from either SIVinfected or control animals at time of sacrifice. The harvested nerve segment was transferred to a two-chamber in vitro recording system that has been described in detail previously. ${ }^{25}$ Inside the bathing chamber of this system, the nerve was superfused with oxygenated synthetic interstitial fluid at physiological $\mathrm{pH}$ and temperature $(\mathrm{pH}$ 7.4 and $32^{\circ} \mathrm{C}$ ). The proximal segment of the nerve was threaded through an opening into a recording chamber filled with paraffin oil. On a dissecting platform inside the recording chamber, the sural nerve was carefully desheathed, and small bundles of the nerve were teased apart using watchmaker forceps. Small filaments were then placed on a silver wire electrode to record extracellularly the neuronal activity from single nerve fibers. The neurophysiological methods used for signal filtering and data recording were described in detail previously. ${ }^{25,26}$ Conduction latencies of nerve fibers were measured in response to a single electrical stimulus (up to $30 \mathrm{~mA}, 0.1 \mathrm{~ms}$ ) applied through a suction electrode, which was placed at the distal end of the nerve inside the bathing chamber. For the calculation of the conduction velocity, the distance between the stimulation and the recording electrode was divided by the measured conduction latency. Nerve fibers with a conduction velocity below $2 \mathrm{~m} / \mathrm{second}$ were classified as unmyelinated C-fibers. ${ }^{26}$ From SIV-infected and uninfected control animals, recordings from 71 and $138 \mathrm{C}$ fibers, respectively, were obtained and analyzed. The experimenters performing the electrophysiological recordings were blind to the SIV infection status of the animal.

\section{Statistical Analysis}

All statistical inferences ( $P$ values) were calculated using parametric methods. Groups of SIV-infected animals and uninfected control animals were compared using oneway analysis of variance (analysis of variance). Subse- 
quent comparisons between any two of the groups used the Bonferroni method to adjust for multiple comparisons. The Pearson correlation coefficient was used to analyze the relationships between continuous variables. To compare C-fiber conduction velocity data between SIV-infected macaques and uninfected control animals, the two-tailed Student's t-test was used. In all analyses, statistical significance was defined as a $P$ value of less than 0.05 .

\section{Results}

\section{Epidermal Nerve Fiber Density Declines with SIV} Infection

Skin biopsies have proven to be an invaluable tool to diagnose small sensory fiber neuropathies, largely replacing sural nerve biopsies for clinical assessments of small fiber sensory neuropathies. ${ }^{8,19,20,27-29}$ Previous studies of skin biopsies taken from HIV-infected patients have shown a close correlation between epidermal nerve fiber (ENF) densities measured at the distal leg region and the presence of neuropathic pain. In those studies, reduced ENF density at the distal leg was associated with increased neuropathic pain, lower CD4 counts, and higher plasma viral loads. ${ }^{29}$ To determine whether epidermal nerve fiber density loss occurred with SIV infection, epidermal nerve fiber densities in footpad samples from SIV-infected macaques were immunostained for PGP9.5, and then were compared with ENF density in uninfected control animals (Figure 1). Immunostaining to detect PGP9.5, a pan-neuronal marker present as a cytoplasmic protein in peripheral nerves, is currently the most consistent method to visualize ENF. ${ }^{30,31}$ A progressive decrease in epidermal nerve fiber density over time developed in SIV-infected macaques resembling ENF decreases reported in HIV-infected individuals. A significant decline in ENF density developed 8 weeks postinoculation and was reduced further at the 12-week postinoculation time point $(P<0.001$; Figure 1).

\section{SIV-Induced Dorsal Root Ganglia Pathology}

The neuropathological changes in the DRG associated with HIV-induced distal sensory polyneuropathy include inflammatory infiltrates of activated macrophages, fewer lymphocytes, decreased numbers of dorsal root ganglion neurons, and the presence of nodules of Nageotte. .,32,33 $^{2}$ To investigate the histopathological lesions in the DRG of SIV-infected macaques, hematoxylin and eosin sections of the fifth lumbar DRG from SIV-inoculated macaques and uninfected controls were examined. DRG from uninfected control macaques did not contain lesions. In contrast, DRG examined from SIV-infected macaques contained multifocal aggregates of infiltrating mononuclear cells including macrophages as well as scattered lymphocytes. Nageotte nodules, with neuronal cell bodies replaced by a mixture of infiltrating mononuclear cells and Schwann cells, and evidence of neuronophagia, with infiltrating macrophages abutting degenerate neurons within the neuronal compartment, were also observed in
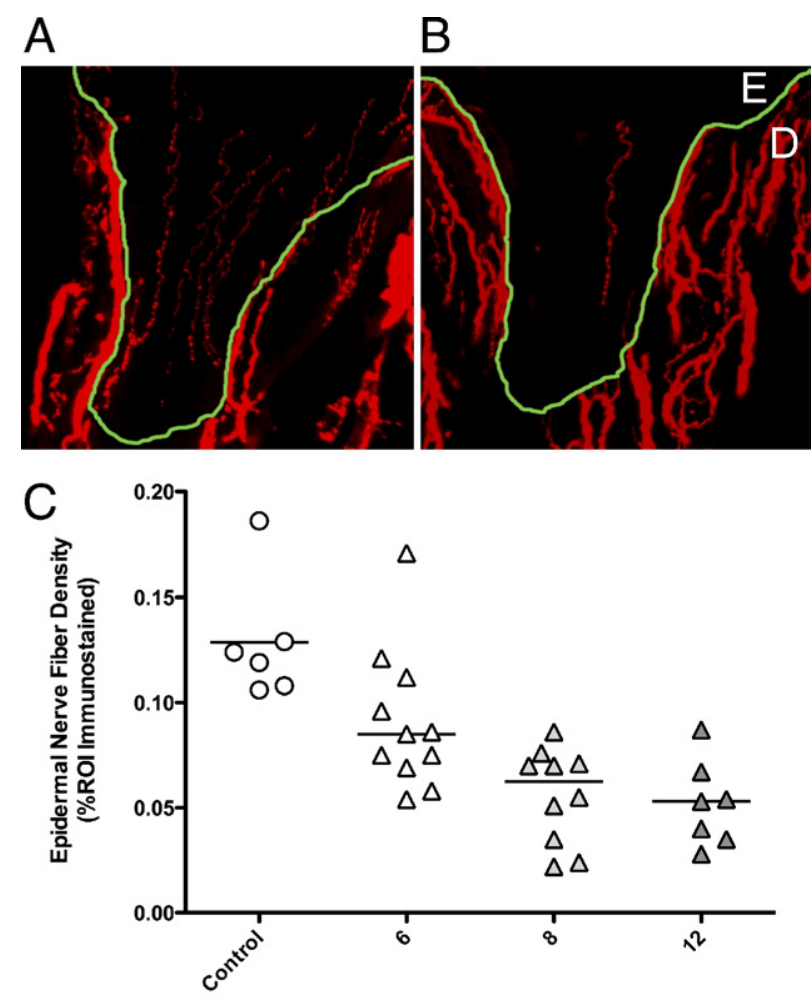

\section{Weeks PI}

Figure 1. Epidermal nerve fiber density is reduced with SIV infection. PGP9.5 immunostained sections of footpad from control (A) and SIV-infected macaques (B) showed a decline in epidermal nerve fiber density. Nerve fibers (red) in skin are present in both epidermis (E), and in dermis (D), with dermal-epidermal junction traced in green. C: Measurement of ENF density in control and SIV-infected macaques demonstrated reduced ENF density in SIV-infected macaques (triangles) compared to uninfected controls (circles), with significant ENF decline beginning 8 weeks p.i., then declining further at the 12 -week time point. $P<0.001$, analysis of variance). PI, postinoculation; ROI, region of interest.

DRG of animals with moderate to severe ganglionitis. These lesions in DRG of SIV-infected macaques demonstrated that SIV infection induces DRG pathology closely resembling the lesions reported in dorsal root ganglia of HIV-infected individuals with distal sensory polyneuropathy. ${ }^{1,4}$ In contrast with DRG lesions, microscopic examination of plastic-embedded, toluidine blue-stained sections of sural nerve and peroneal nerves did not reveal evidence of neuritis or damage to myelinated fibers in SIV-infected macaques despite the presence of inflammatory changes in the lumbar DRG. Inflammatory lesions may still be present multifocally in peripheral nerve but may be difficult to detect given the extensive length of peripheral nerves. A similar paucity of lesions in sural nerve has been reported in HIV neuropathy. ${ }^{34,35}$

\section{Dorsal Root Ganglia Macrophage and Satellite Cell Activation in SIV-Infected Macaques}

Macrophage infiltration has been reported in previous immunopathological studies performed on DRG from HIV-infected patients with distal sensory polyneuropathy as well as in trigeminal ganglia in SIV-infected macaques. $^{14,32}$ To investigate whether a similar process 


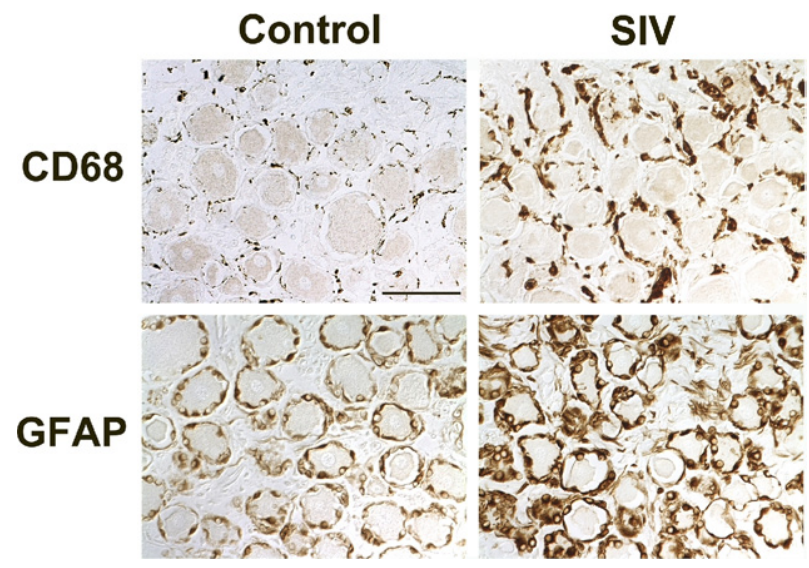

Figure 2. Increased CD68 and GFAP expression in DRG of SIV-infected macaques. Immunostaining for the macrophage marker CD68 (top panels) and the satellite cell marker GFAP (bottom panels) in the lumbar DRG was increased in SIV-infected macaques versus uninfected control animals. Sections of DRG from control macaques contained scattered CD68-positive resident macrophages diffusely distributed in the perineuronal compartment, whereas SIV-infected macaques had increased immunostaining for CD68 both in cells consistent with infiltrating macrophages and diffusely in activated, endogenous macrophages in DRG. Scale bar $=50 \mu \mathrm{m}$.

developed in macaque DRG following SIV infection, mononuclear cell populations in the DRG from control macaques and SIV-infected macaques were characterized by immunostaining DRG sections for the macrophage marker CD68 (Figure 2). A resident population of CD68-positive macrophages was observed in the perineuronal compartment of uninfected control macaques. In SIV-infected macaques, this population of perineuronal endogenous macrophages stained much more intensely for CD68 (Figure 2). Although this immunostaining pattern likely represents activation of endog- enous macrophages, there are currently no immunomarkers that allow infiltrating macrophages to be distinguished from activated resident macrophages. The amount of CD68 immunostaining was measured by quantitative image analysis as described previously. ${ }^{17}$ Immunostaining for CD68-positive cells in the DRG was significantly higher in SIV-infected macaques than control macaques beginning 6 weeks p.i. $(P=0.008)$. Significant elevation in CD68 expression in the DRG was maintained at both 8 and 12 weeks p.i. (Figure 2).

To determine whether satellite cells were immune activated by SIV infection, DRG sections were immunostained for GFAP followed by measurement of GFAP expression by image analysis (Figure 2). GFAP expression was significantly higher in DRG of SIV-infected animals 6 weeks p.i., and these elevated GFAP levels were maintained at 8 weeks p.i. (Figure 3B) before declining to control animal levels at 12 weeks p.i. This pattern of up-regulation resembled sustained increases in CD68 expression in the DRG at both 6 and 8 weeks p.i.; however, CD68 remained elevated at 12 week p.i., unlike GFAP. To determine how stable SIV replication levels were in the DRG throughout infection, SIV RNA levels were measured by real-time RT-PCR (Figure 3D). The amount of SIV RNA in DRG was relatively constant from 6 weeks p.i. through 12 weeks p.i., demonstrating that SIV replication in DRG continues throughout infection.

\section{SIV Infects Macrophages in Dorsal Root Ganglia}

Previous studies have shown virus mostly localized in perivascular inflammatory cells and the nodules of $\mathrm{Na}$ geotte in the DRG of patients with HIV neuropathy. ${ }^{1,4,32}$ To confirm that macrophages were the predominant cell
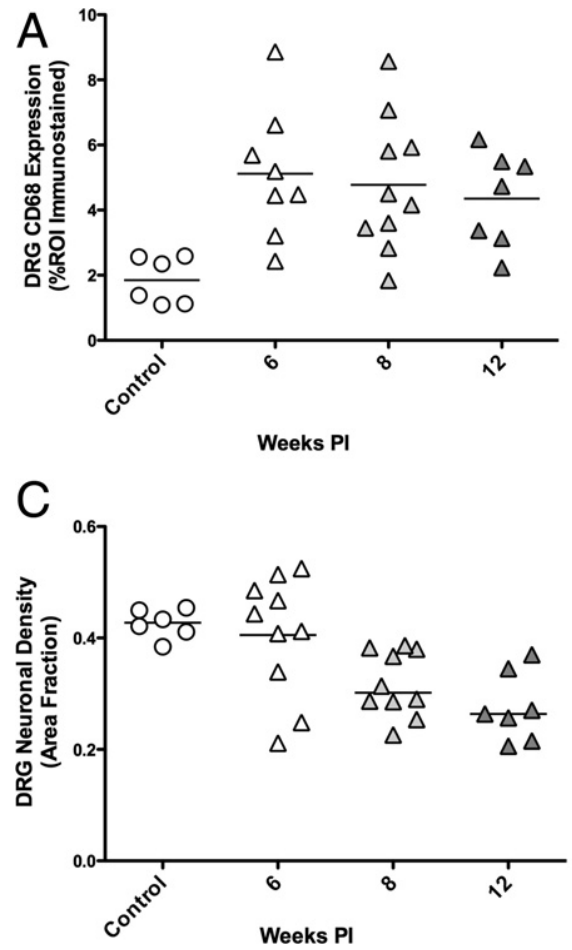

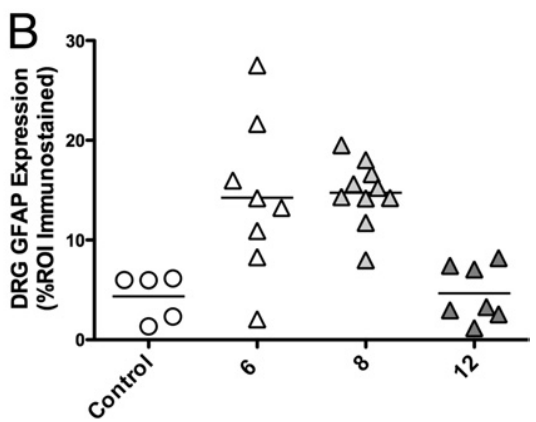

Weeks PI

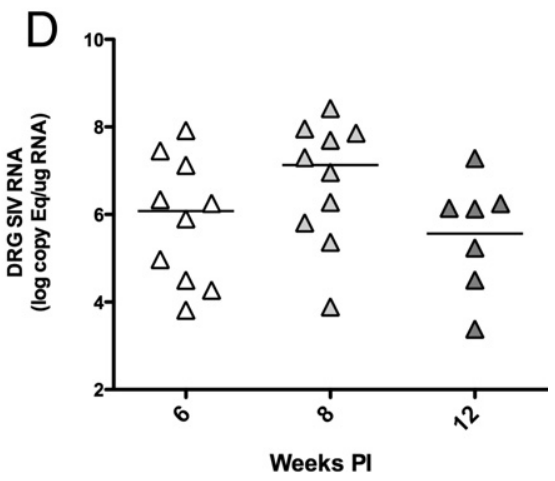

Figure 3. Alterations in CD68, GFAP, neuronal density, and SIV RNA in DRG over time. A: Scatter plot depicting the amount of CD68 immunostaining for macrophages in the dorsal root ganglia of control macaques (circles) and SIV infected macaques (triangles) demonstrates a statistically significant increase in macrophage activation and infiltration in the DRG of SIV infected macaques beginning 6 weeks p.i. and then sustained at comparable levels throughout the course of infection to 12 weeks p.i. $(P=$ 0.008 , analysis of variance). B: A similar significant increase in GFAP immunostaining of satellite cells was measured 6 weeks p.i. as well as 8 weeks p.i., before declining to control animal levels 12 weeks. p.i. $(P=0.001$, analysis of variance). C: Neuronal area fraction measurements in the dorsal root ganglia of control macaques and SIV-infected macaques demonstrated a statistically significant decrease in neuronal density in the dorsal root ganglia of SIV-infected macaques 12 weeks p.i. as compared to control macaques $(P=0.001$, analysis of variance,). D: SIV RNA levels in DRG were stable from 6 to 12 weeks p.i., consistent with sustained CD68 expression over time. Bars represent group means. PI, postinoculation; ROI, region of interest. 


\section{Iba-1}

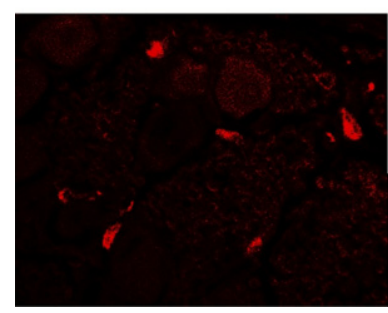

SIV

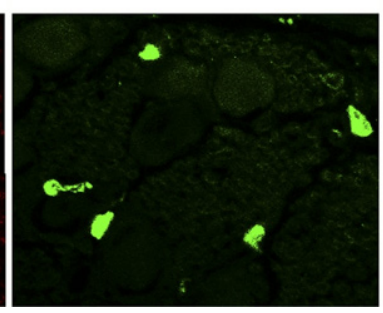

Iba-1/SIV

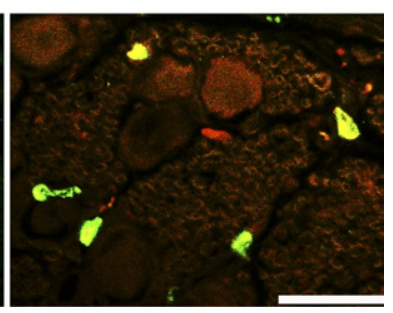

Figure 4. SIV infection of macrophages in the DRG. Confocal laser scanning microscopy was performed on sections of dorsal root ganglia from SIV-infected macaques double immunostained for the macrophage marker Iba-1 (red, left) and SIV gp41 (green, middle). A merged image showed colocalization of Iba-1 and SIV gp41 (yellow, right), indicating that macrophages were the predominant SIV-infected cell type in sensory ganglia. type infected with SIV in DRG, sections were double immunostained for SIV gp41 and the macrophage marker Iba-1, and then examined by laser confocal microscopy. Colocalization of SIV gp41 within macrophages demonstrated that macrophages were clearly the predominant cell type harboring SIV in the DRG (Figure 4).

\section{Reduced DRG Neuronal Density with SIV Infection}

The main morphological features of DRG pathology induced by HIV include neuronal injury and axonal degeneration. Relatively few reports have demonstrated neuronal loss in the DRG in addition to injury as DRG are infrequently examined in patients dying of HIV-induced DSP. ${ }^{13,14,33,36}$ To determine whether neuronal loss developed in DRG of SIV-infected macaques, the neuronal density in DRG was measured using the area fraction fractionator technique, an unbiased stereological method that we have used in previous studies to estimate neuronal density in the trigeminal ganglia of macaques. ${ }^{15}$ These measurements revealed a significant decrease in the fractional area occupied by neurons in the DRG of SIV-infected macaques 12 weeks p.i. as compared to control macaques, consistent with a decrease in the DRG neuronal density $(P=0.001$, Figure $3 C)$.

\section{SIV Induces Functional Changes in C-Fibers in Peripheral Nerves}

Because HIV neuropathy is suspected to principally involve small sensory fibers (ie, small, unmyelinated C-fibers), this markedly limits the value of standard clinical electrophysiological studies for evaluating nerve conduction, both in HIV-infected patients and in SIV-infected macaques, because such assessments predominantly evaluate the conductive properties of large sensory fibers. We therefore used a teased fiber technique that allows single nerve fiber activity to be recorded from unmyelinated nerve fibers to measure conduction velocity specifically in these fibers. C-fiber conduction velocity studies showed that significant decline in C-fiber conduction velocity in SIV-infected macaques developed 12 weeks p.i. ( $P=0.0003$, Figure $5 \mathrm{~A})$. C-fiber conduction velocity measurements from SIV-infected macaques (71 total recordings) or from control animals (138 total recordings) were pooled, and then separated into bins (bin size of $0.1 \mathrm{~m} /$ second) to generate a histogram allowing comparison of C-fiber conduction in SIV-infected macaques 12 weeks postinfection versus the uninfected control macaques (Figure 5B). The percentage of fibers with conduction velocities below $0.5 \mathrm{~m} /$ second increased in SIV-infected animals, whereas the percentage of fibers with conduction velocities $>0.5 \mathrm{~m} / \mathrm{second}$ decreased as compared to uninfected control animals. Overall, C-fiber conduction velocities in SIV-infected animals showed a clear leftward shift to lower conduction velocity values 12 weeks postinoculation with SIV $(P<0.001$; Student's t-test).

\section{Decreased C-Fiber Conduction Velocity is Highly Correlated with SIV-Induced DRG Pathology}

To determine whether SIV-induced alterations in peripheral nerve function were related to DRG changes, the
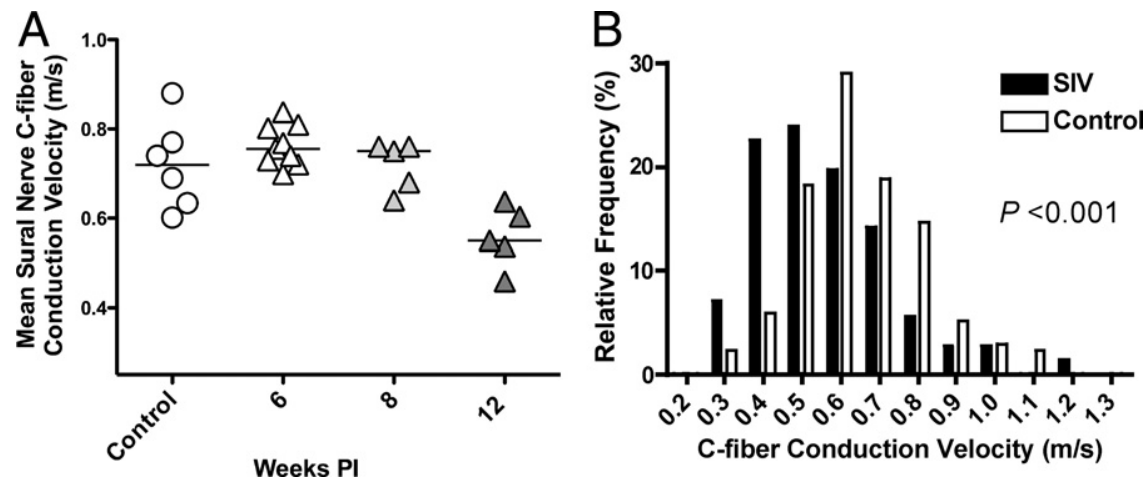

Figure 5. Decreased C-fiber conduction velocity in SIV-infection. A: Mean sural nerve C-fiber conduction velocity for the group of SIV-infected animals evaluated 12 weeks p.i. was significantly slower than for uninfected control animals as well as for the groups of SIV-infected animals evaluated 6 and 8 weeks postinoculation $(P=0.003$, analysis of variance). $\mathbf{B}$ : To characterize the functional alterations in peripheral nerves that developed with SIV infection, the conduction velocities of $\mathrm{C}$-fibers in sural nerves from SIV-infected monkeys collected 12 weeks p.i. were compared with the conduction velocities in uninfected control animals. The histogram containing the pooled C-fiber CV measurements for SIV-infected (black bars) and control macaques (white bars) showed an increase in the percentage of nerve fibers with slower conduction velocities in SIV-infected macaques 12 weeks p.i. as compared to controls $(P<$ 0.001 ; Student's $t$-test). PI, postinoculation. 


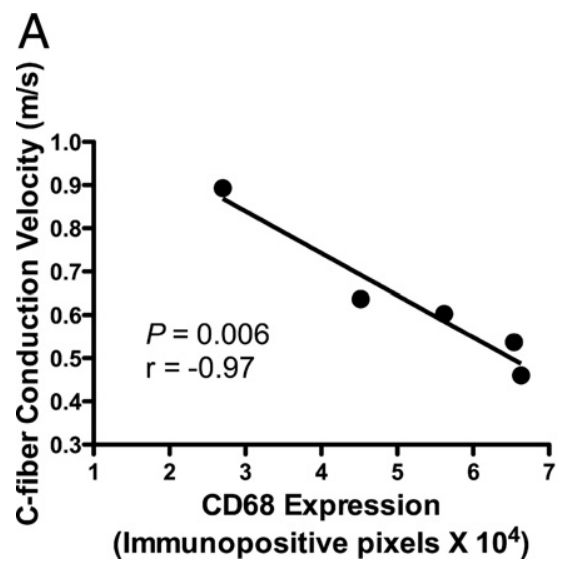

mean C-fiber conduction measurements of SIV-infected animals 12 weeks p.i. were compared with lumbar DRG CD68 expression and DRG neuronal density (Figure 6). The mean C-fiber CV of SIV-infected animals was strongly inversely correlated with macrophage infiltration $(P=0.006, r=-0.97$; Figure $6 \mathrm{~A})$ and highly correlated with DRG neuronal density $(P=0.008, r=0.96$; Figure $6 \mathrm{~B})$. In contrast, C-fiber CV decline was not well correlated with the amount of SIV RNA in the DRG $(P=0.71$, $r=0.22)$. These findings indicated that the extent of macrophage activation and infiltration in the DRG corresponded closely with functional changes in C-fiber conduction properties. In contrast to the relationship between CV slowing and extent of CD68 expression in DRG, slowing of $\mathrm{C}$-fiber $\mathrm{CV}$ was not strongly correlated with the extent of ENF loss ( $P=0.43, r=0.46)$ suggesting that although HIV and SIV clearly induce damage to the distal elements of the somatosensory pathway, including loss of terminal C-fiber endings in the epidermis, ENF loss may be secondary to damage to proximal pathway components including cell bodies of sensory neurons located in lumbar DRG.

\section{Discussion}

This study examined the relationship between SIV-induced pathological alterations in key sensory pain pathway components at the level of both the lumbar dorsal root ganglia and the terminal epidermal nerve fibers in the skin and the associated functional changes in the conduction properties of the small C-fibers located in the sural nerve that transmit information from epidermal nociceptors to the DRG. SIV-induced changes in the lumbar dorsal root ganglia, including both the amount of macrophage infiltration and the extent of decline in neuronal density, were strongly correlated with decreases in C-fiber conduction velocity. The close relationship between SIV-induced DRG damage and decreased conduction velocity in C-fibers supports the hypothesis that HIVinduced functional change in unmyelinated nerve fibers is the result of damage to the sensory nerve cell bodies located in the DRG. The lack of inflammatory lesions in sural nerve despite decline in C-fiber conduction velocity further emphasizes that SIV-induced damage at the level of the DRG may be responsible for altered C-fiber conduction properties. Future studies aimed at characterizing damage to and loss of specific subsets of DRG neurons including the small-diameter nociceptive neuronal cell bodies associated with C-fibers will be necessary to determine whether $\mathrm{C}$-fiber-associated neurons in DRG are particularly susceptible in HIV/SIV infection.

Decreases in nerve fiber conduction velocity have been reported in both rat and macaque models of diabetic neuropathy. ${ }^{37,38}$ Similarly, our studies have demonstrated a decrease in C-fiber conduction velocity in SIVinfected macaques during late stage infection. Routine clinical electrophysiological assessments of nerve conduction velocity performed on HIV-infected patients with neuropathy have not been found to be of significant diagnostic value probably because current standard clinical electrophysiological evaluations are not sensitive enough to measure C-fiber conduction velocities apart from myelinated fibers. ${ }^{39}$ Rather, ex vivo recordings of peripheral nerve containing C-fibers, as performed in this study, are the only current practical means of measuring C-fiber conduction velocities. Furthermore, compound action potential recordings are not easily standardized and interpreted, since a loss of amplitude of the C-fiber wave can be either due to a loss of nerve fibers or a decrease in conduction velocity. To evaluate C-fiber conduction, this study used the teased fiber technique to record activity from single nerve fibers.

Although diabetic neuropathy and HIV-induced neuropathy differ in etiology, the pathogenesis of these processes may converge at the point of alterations in ionic conductance including expression or distribution of voltage-gated sodium and/or potassium channels. Despite a great interest in sodium channel biology in studies of diabetic neuropathy and other painful neuropathies such as erythromelalgia, no studies to date have examined the role of voltage-gated sodium channels in HIV neuropathy. ${ }^{40}$ The SIV/macaque model of PNS disease is well suited for such studies because physiological alterations in nerve fiber conduction properties can be evaluated in association with corresponding DRG neuron damage and loss, SIV replication, host inflammatory responses, and concurrent alterations in voltage-gated channel expression in the PNS. 
Dorsal root ganglia have been infrequently examined in patients autopsied with HIV/AIDS; however, described neuropathological changes include increased macrophage infiltration, decreased numbers of DRG neurons, the presence of Nageotte nodules, and virus replication in macrophages. ${ }^{4,7}$ These changes in DRG of HIV-infected individuals parallel our observations of lumbar DRG alterations developing in SIV-infected macaques. In both the PNS and central nervous system, viral-induced damage to neurons appears to be an indirect phenomenon as neurons are not productively infected. Rather, neurotoxic products produced by activated macrophages and viral proteins may contribute synergistically to neuronal damage. ${ }^{41,42}$ Because replication of SIV in DRG macrophages may be the initiating, primary cause of DRG damage, therapeutic strategies aimed at tightly controlling viral replication and associated inflammatory responses may aid HIV-infected individuals suffering from HIV-induced peripheral neuropathy.

In agreement with studies demonstrating ENF declines in skin biopsies from HIV-infected individuals with neuropathy, we also detected a reduction in ENF densities in plantar skin punches from SIV-infected macaques when compared to uninfected animals. ${ }^{19,29}$ Alterations in ENF density are consistent with our report of impaired ENF regeneration postaxotomy in SIV-infected macaques. ${ }^{43}$ In nerves, SIV-induced damage is first apparent in the most distal innervation territory (skin) and only at later stages of the disease at more proximal sites (sural nerve). Our finding that a decrease in epidermal nerve fiber density develops before decline in nerve conduction velocity demonstrates that longer fibers are more sensitive to SIV-induced damage. Thus, the lack of correlation between ENF loss and CV slowing likely reflects differences in sampling sites.

In this study, to measure epidermal nerve fiber density in the most distal sensory regions, skin samples were harvested from the glabrous (non-haired) skin of the plantar footpad of SIV-infected macaques. Skin biopsy samples from humans, in contrast, are generally harvested from haired skin at the level of the lateral malleolus, a more proximal site. Having noted that epidermal nerve fiber density is much higher in macaque glabrous skin than in human haired skin, we used a collapsed Z-stack technique to measure PGP9.5 immunostaining in epidermis rather than counting the number of PGP9.5 immunopositive epidermal nerve fibers, the technique described for HIV-infected individuals. ${ }^{30}$

At this time, the temporal progression of HIV-induced peripheral neuropathy remains poorly defined. Our studies demonstrate that significant inflammatory alterations develop in the DRG by 6 weeks postinoculation with SIV, corresponding with abundant SIV replication in the DRG. In previous studies, we have shown SIV RNA in trigeminal ganglia during acute infection 7 days postinoculation, suggesting that DRG also may harbor replicating SIV at early stages of infection. ${ }^{15}$ Significant decline in epidermal nerve fiber density noted at 8 weeks p.i. lagged DRG changes by 2 weeks. Although median ENF density was lower 6 weeks p.i., this decline in SIV-infected macaques versus uninfected control animals was not significant.
Together, these findings show that sensory ganglia are early targets of SIV with potential for inducing early and sustained damage to sensory neurons, especially small neurons and their associated C-fibers. At 12 weeks p.i., C-fiber conduction velocity in sural nerves was significantly lower than earlier time points, and the magnitude of decline correlated strongly with extent of DRG macrophage infiltration. The finding that injury to neurons in the DRG that was mediated by activated macrophages preceded altered conduction of unmyelinated nerve fibers in SIV-infected macaques suggests that macrophage-mediated DRG damage may be the initiating event in HIVinduced sensory neuropathy.

This study establishes the value of the SIV/macaque model for elucidating the pathogenesis of HIV PNS disease, demonstrating that multiple components of the sensory pain pathway can be examined to define the relationship between pathological and functional alterations. Future studies in the SIV/macaque model can facilitate discovery of the molecular mechanisms underlying the functional disturbances caused by HIV in the peripheral nervous system, thereby guiding rational new therapeutic strategies.

\section{Acknowledgments}

We thank Christine Brennan, Suzanne E. Queen, John Anderson, Brandon Dearman, Pat Wilcox, and Bruce Baldwin for excellent technical assistance.

\section{References}

1. McArthur JC, Brew BJ, Nath A: Neurological complications of HIV infection. Lancet Neurol 2005, 4:543-555

2. Ellis RJ, Rosario D, Clifford DB, McArthur JC, Simpson D, Alexander T, Gelman BB, Vaida F, Collier A, Marra CM, Ances B, Atkinson JH, Dworkin RH, Morgello S, Grant I: Continued high prevalence and adverse clinical impact of human immunodeficiency virus-associated sensory neuropathy in the era of combination antiretroviral therapy: the CHARTER Study. Arch Neurol 2010, 67:552-558

3. Cornblath DR, McArthur JC: Predominantly sensory neuropathy in patients with AIDS and AIDS-related complex. Neurology 1988, 38: 794-796

4. Pardo CA, McArthur JC, Griffin JW: HIV neuropathy: insights in the pathology of HIV peripheral nerve disease. J Peripher Nerv Syst 2001 , 6:21-27

5. Simpson DM, Tagliati M: Neurologic manifestations of HIV infection Ann Intern Med 1994, 121:769-785

6. Pettersen JA, Jones G, Worthington C, Krentz HB, Keppler OT, Hoke A, Gill MJ, Power C: Sensory neuropathy in human immunodeficiency virus/acquired immunodeficiency syndrome patients: protease inhibitor-mediated neurotoxicity. Ann Neurol 2006, 59:816-824

7. Brinley FJ Jr., Pardo CA, Verma A: Human immunodeficiency virus and the peripheral nervous system workshop. Arch Neurol 2001, 58:1561-1566

8. Morgello S, Estanislao L, Simpson D, Geraci A, DiRocco A, Gerits P Ryan E, Yakoushina T, Khan S, Mahboob R, Naseer M, Dorfman D, Sharp V: HIV-associated distal sensory polyneuropathy in the era of highly active antiretroviral therapy: the Manhattan HIV Brain Bank. Arch Neurol 2004, 61:546-551

9. Schifitto G, McDermott MP, McArthur JC, Marder K, Sacktor N, Epstein L, Kieburtz K: Incidence of and risk factors for HIV-associated distal sensory polyneuropathy. Neurology 2002, 58:1764-1768

10. Keswani SC, Polley M, Pardo CA, Griffin JW, McArthur JC, Hoke A: Schwann cell chemokine receptors mediate HIV-1 gp120 toxicity to sensory neurons. Ann Neurol 2003, 54:287-296 
11. Bradley WG, Shapshak P, Delgado S, Nagano I, Stewart R, Rocha B: Morphometric analysis of the peripheral neuropathy of AIDS. Muscle Nerve 1998, 21:1188-1195

12. Watkins LR, Goehler LE, Relton J, Brewer MT, Maier SF: Mechanisms of tumor necrosis factor-alpha (TNF-alpha) hyperalgesia. Brain Res 1995, 692:244-250

13. Kennedy JM, Hoke A, Zhu Y, Johnston JB, van Marle G, Silva C, Zochodne DW, Power C: Peripheral neuropathy in lentivirus infection: evidence of inflammation and axonal injury. AIDS 2004, 18:12411250

14. Zhu Y, Jones G, Tsutsui S, Opii W, Liu S, Silva C, Butterfield DA, Power C: Lentivirus infection causes neuroinflammation and neurona injury in dorsal root ganglia: pathogenic effects of STAT-1 and inducible nitric oxide synthase. J Immunol 2005, 175:1118-1126

15. Laast VA, Pardo CA, Tarwater PM, Queen SE, Reinhart TA, Ghosh M Adams RJ, Zink MC, Mankowski JL: Pathogenesis of simian immunodeficiency virus-induced alterations in macaque trigeminal ganglia. J Neuropathol Exp Neurol 2007, 66:26-34

16. Zink MC, Suryanarayana K, Mankowski JL, Shen A, Piatak M Jr, Spelman JP, Carter DL, Adams RJ, Lifson JD, Clements JE: High vira load in the cerebrospinal fluid and brain correlates with severity of simian immunodeficiency virus encephalitis. J Virol 1999, 73:1048010488

17. Mankowski JL, Clements JE, Zink MC: Searching for clues: tracking the pathogenesis of human immunodeficiency virus central nervous system disease by use of an accelerated, consistent simian immunodeficiency virus macaque model. J Infect Dis 2002, 186 (Suppl 2):S199-208

18. Zink MC, Laast VA, Helke KL, Brice AK, Barber SA, Clements JE, Mankowski JL: From mice to macaques-animal models of HIV nervous system disease. Curr HIV Res 2006, 4:293-305

19. Holland NR, Stocks A, Hauer P, Cornblath DR, Griffin JW, McArthur JC: Intraepidermal nerve fiber density in patients with painful sensory neuropathy. Neurology 1997, 48:708-711

20. McCarthy BG, Hsieh ST, Stocks A, Hauer P, Macko C, Cornblath DR Griffin JW, McArthur JC: Cutaneous innervation in sensory neuropathies: evaluation by skin biopsy. Neurology 1995, 45:18481855

21. Nolano M, Provitera V, Crisci C, Stancanelli A, Wendelschafer-Crabb G, Kennedy WR, Santoro L: Quantification of myelinated endings and mechanoreceptors in human digital skin. Ann Neurol 2003, 54:197205

22. Vargas DL, Nascimbene C, Krishnan C, Zimmerman AW, Pardo CA Neuroglial activation and neuroinflammation in the brain of patients with autism. Ann Neurol 2005, 57:67-81

23. West MJ, Coleman PD, Flood DG, Troncoso JC: Differences in the pattern of hippocampal neuronal loss in normal ageing and Alzheimer's disease. Lancet 1994, 344:769-772

24. Kril JJ, Patel S, Harding AJ, Halliday GM: Neuron loss from the hippocampus of Alzheimer's disease exceeds extracellular neurofibrillary tangle formation. Acta Neuropathol (Berl) 2002, 103:370-376

25. Ali Z, Ringkamp M, Hartke TV, Chien HF, Flavahan NA, Campbell JN Meyer RA: Uninjured C-fiber nociceptors develop spontaneous activity and alpha-adrenergic sensitivity following L6 spinal nerve ligation in monkey. J Neurophysiol 1999, 81:455-466

26. Wu G, Ringkamp M, Hartke TV, Murinson BB, Campbell JN, Griffin JW, Meyer RA: Early onset of spontaneous activity in uninjured C-fiber nociceptors after injury to neighboring nerve fibers. J Neurosci 2001, 21:RC140
27. Herrmann DN, McDermott MP, Henderson D, Chen L, Akowuah K, Schifitto G: Epidermal nerve fiber density, axonal swellings and QST as predictors of HIV distal sensory neuropathy. Muscle Nerve 2004 29:420-427

28. Herrmann DN, Griffin JW, Hauer P, Cornblath DR, McArthur JC: Epidermal nerve fiber density and sural nerve morphometry in peripheral neuropathies. Neurology 1999, 53:1634-1640

29. Polydefkis M, Yiannoutsos CT, Cohen BA, Hollander H, Schifitto G, Clifford DB, Simpson DM, Katzenstein D, Shriver S, Hauer P, Brown A, Haidich AB, Moo L, McArthur JC: Reduced intraepidermal nerve fiber density in HIV-associated sensory neuropathy. Neurology 2002, 58 : 115-119

30. Kennedy WR, Wendelschafer-Crabb G, Johnson T: Quantitation of epidermal nerves in diabetic neuropathy. Neurology 1996, 47:10421048

31. Horiuchi Y, Bae S, Katayama I: Nerve growth factor (NGF) and epidermal nerve fibers in atopic dermatitis model NC/Nga mice. J Dermatol Sci 2005, 39:56-58

32. Keswani SC, Pardo CA, Cherry CL, Hoke A, McArthur JC: HIVassociated sensory neuropathies. AIDS 2002, 16:2105-2117

33. Esiri MM, Morris CS, Millard PR: Sensory and sympathetic ganglia in HIV-1 infection: immunocytochemical demonstration of HIV-1 vira antigens, increased $\mathrm{MHC}$ class II antigen expression and mild reactive inflammation. J Neurol Sci 1993, 114:178-187

34. Gonzalez-Duarte A, Cikurel K, Simpson DM: Managing HIV peripheral neuropathy. Curr HIV/AIDS Rep 2007, 4:114-118

35. Gonzalez-Duarte A, Robinson-Papp J, Simpson DM: Diagnosis and management of HIV-associated neuropathy. Neurol Clin 2008, 26 : 821-832

36. Jones G, Zhu Y, Silva C, Tsutsui S, Pardo CA, Keppler OT, McArthur JC, Power C: Peripheral nerve-derived HIV-1 is predominantly CCR5dependent and causes neuronal degeneration and neuroinflammation. Virology 2005, 334:178-193

37. Price SA, Agthong S, Middlemas AB, Tomlinson DR: Mitogen-activated protein kinase $\mathrm{p} 38$ mediates reduced nerve conduction velocity in experimental diabetic neuropathy: interactions with aldose reductase. Diabetes 2004, 53:1851-1856

38. Cornblath DR, Hillman MA, Striffler JS, Herman CN, Hansen BC: Peripheral neuropathy in diabetic monkeys. Diabetes 1989, 38:13651370

39. Hahn K, Arendt G, Braun JS, von Giesen HJ, Husstedt IW, Maschke M, Straube ME, Schielke E: A placebo-controlled trial of gabapentin for painful HIV-associated sensory neuropathies. J Neurol 2004, 251 : $1260-1266$

40. Keswani SC, Hoke A: Incidence of and risk factors for HIV-associated distal sensory polyneuropathy, Neurology 2003, 61:279; author reply $279-280$

41. Babas T, Munoz D, Mankowski JL, Tarwater PM, Clements JE, Zink MC: Role of microglial cells in selective replication of simian immunodeficiency virus genotypes in the brain. J Virol 2003, 77:208-216

42. Mankowski JL, Queen SE, Tarwater PM, Fox KJ, Perry VH: Accumulation of beta-amyloid precursor protein in axons correlates with CNS expression of SIV gp41. J Neuropathol Exp Neurol 2002, 61:85-90

43. Ebenezer GJ, Laast VA, Dearman B, Hauer P, Tarwater PM, Adams RJ, Zink MC, McArthur JC, Mankowski JL: Altered cutaneous nerve regeneration in a simian immunodeficiency virus/macaque intracutaneous axotomy model. J Comp Neurol 2009, 514:272-283 